\title{
The application research of Coal seam CO source identification based on the D-S evidence conflict
}

\author{
Nan Feng ${ }^{a}$, Li Yang \\ Hebei Polytechnic University, China \\ abluenanfengly@163.com, ${ }^{b}$ liyang@heuu.edu.cn
}

Keywords: Dempster-Shafter fusion; K-L information distance; conflict identification

\begin{abstract}
In the paper, we introduce the distance function of K-L information to replace the conflict coefficient in order to solve the constraint problem of D-S evidence theory in information fusion and achieve the feature recognition aggreation of Multi-source information under the conflict condition. Simulating results show that: the effective conflict identification of K-L information distance can complete the application constraint of D-S theory's synthetic rule, obtaining the optimized convergence results for synthesis of normal conflicting evidences, isolating the highly conflicting evidence.
\end{abstract}

\section{Introduction}

Along with the coal mine information technology advancing, using wireless sensor to CO gas monitoring can provide accurate data parameters, but at the same time increasing the false alarm rate, and thus causing economic loss of false alarm cut-off and excessive preventing; In the underground complex environment and all kinds of uncertain factors, taking $\mathrm{CO}$ as an independent monitoring indicators can not provide accurate information, and thus false alarms can make threat to the production personnel and property safety.Using multi sensor as the hardware foundation, founding multi-source information fusion technology platform, introducing parameter characteristics analysis of CO source for recognition based on the D-S evidence theory can provide reliable technical method and decision-making basis for the coal mine safety early warning system.

Dempster-Shafer (DS) theory of evidence from the 1960s multifunctions has the inference ability with uncertain information and shows good practical performance on data fusion and information prediction. Using multiple evidence information mutual support and complementary advantages, Dempster-Shafer ( D-S ) evidence theory can improve the accuracy of the information synthesis. However in reality because of the environmental complexity, there is different levels of conflict ( information inconsistent ) between evidence, causing D-S evidence theory application defects. Zadeh $^{[1]}$ proposed the conflict in D-S evidence theory firstly, Yager ${ }^{[2]}$ believes that all conflict is unable to provide useful information, Lefever, et $\mathrm{al}^{[3]}$ argue that the conflict is a kind of information. On the premise of confirming the value of conflict information, introducing the K-L information distance function to replace traditional D-S conflict coefficient $\mathrm{K}$, defining K-L evidence conflict degree to determine the conflict characteristic, combining with the low temperature oxidation mechanism of coal spontaneous combustion and primary occurrence characteristics of $\mathrm{CO}$ can discern the source of detected CO In the mine.

\section{D-S Evidence Theory and Evidence Conflict}

\subsection{Preliminaries of the D-S evidence theory}

The D-S theory assumes a non-empty set $\Theta$, to describe all elements with mutually exclusive and exhaustive in the constitute space, $\Theta=\left\{A_{1}, A_{2} \cdots A_{N}\right\}$ called the frame of discernment. Element is a collection of $\mathrm{N}$ mutually exclusive $2^{N}$ subset, denoted by $2^{\ominus}$, quality of evidence (Mass) is the trust to support the proposition in DS theory. 
Definition $\mathbf{1}^{[1]}$ Set $\Theta$ is the identification of D-S theory framework, comprehensive and mutually exclusive propositions composed of power set function $m: 2^{\ominus} \rightarrow[0,1]$, and suffice

$$
m(\Phi)=0, \quad \sum_{A \subset \Theta} m(A)=1
$$

$m(A)$ is the basic probability assignment function under the framework $\Theta$ of $\mathrm{A}$, On behalf of the trust and support of evidence, While $m(A) \neq 0$, A is the focal element of BPA (focus on evidence A).

Definition $2^{[1]}$ Support or belief function(bel) on behalf of the total trust to proposition A with the decision-makers,

$$
\operatorname{Bel}(A)=\sum_{B \subseteq A} m(B), \quad A \subseteq \Theta, B \neq \Phi
$$

Belief function is applied to a collection and all subsets, Bel-based on the global trust.

D-S theoretical composition rules:

Evidences M1、M2 in one framework $\Theta$,

$\mathrm{BPA} \rightarrow \mathrm{m} 1 、 \mathrm{~m} 2$, the focal elements are $\left(A_{1}, \cdots, A_{k}\right)\left(B_{1}, \cdots, B_{l}\right)$, assumes:

$$
K=\sum_{A_{i 1} \cap B_{j 2}=\Phi} m_{1}\left(A_{i}\right) m_{2}\left(B_{j}\right)
$$

$\mathrm{K}$ is the conflict coefficient, characterization the degree of contradictions between Evidences in combination of D-S.

Evidence Fusion results:

$$
\begin{aligned}
m(C) & =\mathrm{m}_{1} \oplus m_{2}=m_{1}(A)+m_{2}(B) \\
& =\frac{1}{1-K} \sum_{A_{1} \cap B_{j 2}=C} m_{1}\left(A_{i}\right) m_{2}\left(B_{j}\right) \quad \forall C \subset \Theta, C \neq \Phi
\end{aligned}
$$

$m(C)$ is the new evidence assignment after the orthogonal combination; $\oplus$ is Orthogonally, (1-K) as the normalization factor which excluding conflict interference.

\subsection{The evidence conflicts's proposed}

1984, Zadch ${ }^{[1]}$ examples demonstrate the drawbacks of the normalization factor, noting the application restrictions under conflict of evidence in Dempster combination rule ${ }^{[4]}$. On the basis of the Zadch, constructed three complex paradox situation by application on the Dempster combination rule, and obtained instance demonstration on Studies of substantial evidence conflict ${ }^{[5,6]}$.

To the conflict coefficient $\mathrm{K}$ :

$\mathrm{K}=1$, evidences $\mathrm{m} 1 、 \mathrm{~m} 2$ are completed conflicts, $\mathrm{D}-\mathrm{S}$ theoretical composition rules fails;

$\mathrm{K}=0$, The evidence information completely consistent, application the Dempster synthesis can achieve fast focus;

$\mathrm{K} \rightarrow 1$, There is a high degree of conflict between the evidences, the fusion result may appear a paradox;

$0<\mathrm{K}<1$, Two evidences partially compatible.

Hence, any evidence $(K \neq 0)$ combination will be results varying degrees of impact due to the existence of the conflict of fusion.To solve the above problems , this paper introduces the K-L information distance express distribution difference between the evidences, trying to provides a viable solution for the evidence theory under the features conflict from independent evidence to the evidence system Perspective.

\section{Expression of the K-L Information Distance in Evidence Conflict}

\subsection{The concept of K-L information distance}

K-L information distance proposed by the statistician Kullback and Leibler in the middle of the 20th century, used to determine the closeness of the two probability distributions. 
Definition $3^{[7]}$ In one framework $\Theta$, the BPA of evidences M1 M2 $\rightarrow m_{1}(x) m_{2}(x)$, information distance from $\mathrm{M} 1$ to $\mathrm{M} 2$ is:

$$
\underset{B P A}{I}\left(m_{1}: m_{2}\right)=\sum_{i=j=1}^{k} m_{1}\left(A_{i}\right) \frac{m_{1}\left(A_{i}\right)}{m_{2}\left(B_{j}\right)}
$$

Definition 3 shows, $I\left(m_{1}: m_{2}\right) \geq 0$, and only $A_{i}=B_{j}$ then $I\left(m_{1}: m_{2}\right)=0$. Greater the K-L information distance, greater the difference of probability assignment function between the two pieces of evidence, but the smaller in the contrary. When the two evidence fully consistent, $\mathrm{I}=0$. Therefore, K-L information distance and D-S conflict coefficient $\mathrm{K}$ has the same meaning, by describing the differences in the distribution of BPA function to reflect the degree of conflict among the evidences.

\subsection{Identifying of evidence conflict by the K- $\mathrm{L}$ information distance}

Evidences of plurality of uniformly distributed (random) in limited temporal, $M_{i}, i=1,2, \cdots, n$. Command

$I_{i, j}=I\left(m_{i}: m_{j}\right)$, generally $I_{i j}\left(m_{i}: m_{j}\right) \neq I_{j i}\left(m_{j}: m_{i}\right)$, then $I_{i j}$ express the information distance from evidence $\mathrm{j}$ to $\mathrm{i}$, structure matrix of K-L information distance:

$$
\left(\begin{array}{ccccc}
I_{11} & I_{12} & \cdots & \cdots & I_{1 n} \\
I_{21} & I_{22} & \cdots & \cdots & I_{2 n} \\
\vdots & \vdots & \vdots & \vdots & \vdots \\
I_{n 1} & I_{n 2} & \cdots & \cdots & I_{n n}
\end{array}\right)
$$

In the selected area, total information distance of all evidences to the evidence is:

$$
I_{i}=\sum_{j=1}^{n} I_{i, j}
$$

Obviously, when $i=j, I_{i j}=0 . I_{i}$ characterized the degree of difference of the distribution function from evidence $i$ to all the evidence in the integration system.

Definition 4 In order to manifest the influence degree of conflict among the evidences in the whole system, through the distribution difference in the proportion of the overall difference, defined distribution difference rate $H_{i}$ is:

$$
H_{i}=\frac{I_{i}}{\sum_{i=1}^{n} I_{i}} \quad i=1,2, \cdots, n .
$$

Formula (6) called $H_{i}$ as evidence conflict degree based on K-L distance information (hereinafter referred to K-L evidence conflict degree). Therefore, K- L evidence conflict degree can representation independent evidence $\mathrm{i}$ and other evidence of the conflict features, also can show the total conflict the influence of position of evidences in the whole system.

\section{Simulation experiment}

In the objective monitoring system of a limited time and space, it is assumed that there is a Identification framework $\Theta=\{A, B, C, \Phi\}=\{$ Combustion, Non spontaneous combustion, uncertain, $\Phi\}$. It is assumed that a system is composed of two groups of multi-sensor monitoring evidence which has three system characteristic parameters: $C O$, Coal mild, Oxygen content.BPA functions $m(A) 、 m(B)$ 和 $m(C)$ are as follows, $m_{i j}(\Phi)=0$ and $\sum_{\bullet \subset \Theta} m_{i}(\bullet)=1$.

Table 1 Analog data (spontaneous combustion) of coal mine safety monitoring system

\begin{tabular}{l|ll|ll|ll}
\hline & \multicolumn{2}{|c}{$\mathrm{m}(\mathrm{A})$} & \multicolumn{3}{c}{$\mathrm{m}(\mathrm{B})$} & \multicolumn{2}{c}{$\mathrm{m}(\mathrm{C})$} \\
\cline { 2 - 6 } & $\mathrm{I}$ & II & I & II & I & II \\
\hline
\end{tabular}




\begin{tabular}{c|c|cc|cc|cc}
\hline CO-BPA & 1 & 0.80 & 0.80 & 0.10 & 0.10 & 0.10 & 0.10 \\
& 2 & 0.75 & 0.75 & 0.15 & 0.15 & 0.10 & 0.10 \\
& 3 & 0.70 & 0.70 & 0.10 & 0.10 & 0.20 & 0.20 \\
$\mathrm{~T}^{\circ} \mathrm{C}$-BPA & 4 & 0.60 & 0.10 & 0.20 & 0.80 & 0.20 & 0.10 \\
& 5 & 0.65 & 0.15 & 0.25 & 0.70 & 0.10 & 0.15 \\
& 6 & 0.55 & 0.20 & 0.15 & 0.75 & 0.30 & 0.05 \\
$\mathrm{O}_{2}$-BPA & 7 & 0.50 & 0.10 & 0.20 & 0.20 & 0.30 & 0.70 \\
& 8 & 0.55 & 0.20 & 0.25 & 0.25 & 0.20 & 0.55 \\
& 9 & 0.55 & 0.15 & 0.30 & 0.30 & 0.15 & 0.55 \\
\hline
\end{tabular}

Establish the K-L distance matrix, by type (4)-( 6) calculated:

Table 2 Much evidence fusion K-L information distance and the degree of evidence conflict

\begin{tabular}{c|ccccccccc}
\hline & 1 & 2 & 3 & 4 & 5 & 6 & 7 & 8 & 9 \\
\hline $\mathrm{I} 1$ & 0.849 & 0.590 & 0.558 & 0.352 & 0.584 & 0.743 & 0.812 & 0.494 & 0.676 \\
$\mathrm{H} 1$ & 0.150 & 0.104 & 0.099 & 0.062 & 0.103 & 0.131 & 0.144 & 0.087 & 0.119 \\
\hline $\mathrm{I} 2$ & 6.891 & 6.021 & 5.673 & 6.249 & 4.847 & 5.735 & 6.820 & 4.643 & 4.802 \\
$\mathrm{H} 2$ & 0.133 & 0.116 & 0.110 & 0.121 & 0.094 & 0.111 & 0.132 & 0.090 & 0.093 \\
\hline
\end{tabular}

In table two, $I_{i j}$ is the K-L information distance from the $\mathrm{j}$ evidence to I evidence, $H_{i}$ is the degree of conflict feature based on the K-L information distance $I_{i}$ in system total gap ratio computational evidence I.

It is a low conflict because of Evidence for 1 group $I_{1 i} \prec 1$ and shows that the feature of monitoring parameter has consistency in the overall system and therefore is applicable to use D-S evidence theory for information fusion; it is a high conflict because of Evidence for 2 groups $I_{2 i} \in(4,7)$ and shows that the feature of monitoring parameter do not have consistency in the overall system and therefore is not applicable to process the data.

According to $I_{i} 、 H_{i}$ comprehensive judgment, we conduct D-S information fusion on the evidence of low conflict 1 group. The data are substituted into the formula (2-3) to obtain the fusion results are as follows:

Table 3 D-S information fusion results of evidence for 1 group

\begin{tabular}{cll}
\hline $\mathrm{D}-\mathrm{S}$ & conflicts coefficient & \multicolumn{1}{c}{ Bel of fusion results } \\
\hline & & $\mathrm{m}_{1}(\mathrm{~A})=0.99998$ \\
$\mathrm{~m}_{11}, \mathrm{~m}_{12}, \cdots, \mathrm{m}_{19}$ & $\mathrm{~K}_{1}=0.450017$ & $\mathrm{~m}_{1}(\mathrm{~B})=1.23841 \mathrm{E}-05$ \\
& & $\mathrm{~m}_{1}(\mathrm{C})=7.9258 \mathrm{E}-06$ \\
& $\mathrm{~m}_{1}(\Theta)=0$ \\
\hline
\end{tabular}

In the figure $3, \mathrm{~m}_{1}(\mathrm{~A})=0.99998 \rightarrow 1$ shows that the mine coal seam spontaneous combustion has occurred somewhere. If the significant feature conflict does not exist between the evidence, there will be a good convergence effect on DS evidence theory of information fusion of multi-source sensor monitoring data.

Because the three types of monitoring information from evidence for 2 group exist significant conflict, it does not comply with the characteristics of coal spontaneous combustion, the $\mathrm{CO}$ can be judged to be native and the mine gas safety work should be done well timely.

\section{Conclusion}

In this paper, based on the platform of a large number of applications of remote sensing technology, combined with the coal spontaneous combustion prediction experiments indicators, using D-S evidence theory to achieve data fusion of multi-source information can improve the alarm accuracy; 
the introduction of the K-L information distance can help us study identification method of system feature conflict and defining feature of the conflict can help us achieve consistency analysis of system information feature. Simulating results show that: Under the condition of information characteristics consistency, we obtain absolute convergence effect on Seam Spontaneous Combustion and under the condition of Subsystem Feature non-uniform , we identify CO source effectively. This study can provide reliable information basis for the managers to take targeted preventive measures and timely treatment under the different $\mathrm{CO}$ source .

\section{References}

[1] Zadeh L A. Review of books: A mathematical theory of evidence[J]. AI Magazine, 1984, $5(3): 81-83$.

[2] Yager R R. On the D-S framework and new combination rules[J]. Information Sciences, 1987, 41(2): $93-138$.

[3] Lefevre E, Colot $\mathrm{O}$, Vannoorenberghe $\mathrm{P}$. Belief functions combination and conflict management $[\mathrm{J}]$.

Information Fusion, 2002, 3(2): 149-162.

[4] Zhang S D, Wang L D. Absurdities about Dempter-Shafer's rule of combination of evidence[J]. Systems Engineering-Theory \& Practice, 1997, 17(5) : 82-85.

[5] Li W Y, Guo K H. Combination rules of D-S evidence theory and conflict problem[J]. Systems Engineering-Theory \& Practice, 2010, 30(8) : 1422-1432.

[6] Liang C Y, Chen Z M, Huang Y Q, et al. A method of dispelling the absurdities of Dempster-Shafer's rule of combination[J]. Systems Engineering-Theory \& Practice, 2005, $25(3): 7-12$.

[7] REN Kai-jun, WU Meng-da, LIU Qi. The combination of prior distributions based on Kullback information[J].Journal of the Academy of Equipment Command \& Technology, 2002, 13(4): 9092. 